\title{
Microbioassay System for an Anti-cancer Agent Test Using Animal Cells on a Microfluidic Gradient Mixer
}

\author{
Shin-ichiro FujII,* Munehisa Uematsu, ${ }^{* *}$ Soichi Yabuki, ${ }^{*}$ Mitsuru Abo, ${ }^{* *}$ Etsuro Yoshimura, ${ }^{* *}$ \\ and Kiichi $\mathrm{SATO}^{* * *}$ \\ *Institute for Biological Resources and Functions, National Institute of Advanced Industrial Science \\ and Technology (AIST), Central 6, 1-1-1 Higashi, Tsukuba, Ibaraki 305-8566, Japan \\ **Graduate School of Agricultural and Life Sciences, The University of Tokyo, \\ 1-1-1 Yayoi, Bunkyo, Tokyo 113-8657, Japan
}

\begin{abstract}
We developed a novel microbioassay system equipped with a gradient mixer of two solutions, and we applied the microfluidic system to an anti-cancer agent test using living animal cells on a microchip. A microchannel for the gradient mixing of two solutions and eight other microchannels for cell assay were fabricated on a poly(dimethylsiloxane) substrate using a soft-lithography method. The functions necessary for this bioassay, i.e., cell culturing, chemical stimulation, cell staining, and fluorescence determination, were integrated into the microfluidic chip. Eight gradient concentrations of the fluorescein solution, ranging from 1 to $98 \mu \mathrm{g} / \mathrm{ml}$, were archived at $0.1 \mu \mathrm{l} / \mathrm{min}$ on a microchip. A stomach cancer cell line was cultured, and a cell viability assay was conducted using 5-Fluorouracil as an anti-cancer agent on the microchip. Cell viability changed according to the estimated concentration of the agent solution. With the microbioassay system, an anti-cancer agent test was conducted using living cells simultaneously in eight individual channels with the gradient concentration of the agent on a microchip.
\end{abstract}

(Received September 9, 2005; Accepted October 28, 2005)

\section{Introduction}

Bioassay and bioanalysis using cultured cells reveal information useful for diagnostics, drug development, and biochemical research. For example, a bioassay, an experiment that uses living things to test the effects of chemicals, is an indispensable technique for drug screening, evaluation of the safety of chemicals, and other basic life-science research. The conventional bioassay, however, involves troublesome handling procedures such as pipetting and requires many valuable cells and reagents. In recent years, micro total analysis systems ( $\mu$ TAS) have been of great interest to biological researchers for cellular analysis because of their many advantages, including a reduction in the consumption of reagents, samples, and cells; short-time analysis; and ease of handling. ${ }^{1,2}$ However, there have been few reports about the scale merit in a microspace, i.e., laminar flow and diffusion, for a bioassay using living cells on a fluidic microchip. ${ }^{3,4}$ It is expected that difficulties in handling procedures such as pipetting will be greatly reduced by making gradient dilutions which take advantage of the merits in a microspace. In a microchip system, scale merits in a microspace, i.e., a short diffusion distance and a rapid and efficient reaction, have been utilized. For example, an extraction and an interfacial reaction have been reported as applications of microchip systems. ${ }^{5-8}$ Moreover, on a microscale, the Reynolds number becomes very low, and laminar flow is facilitated. The principle of laminar flow has also been applied to particle partition and cell sorting. ${ }^{9,10}$

The conventional microchip, however, is troublesome when it comes to the fabrication of multilayer and cells chamber. We have already reported a polymer-based microanalytical system that allows the microfluidic analysis and easy fabrication of a microchip. ${ }^{11,12}$ We have also reported a microbioassay system using suspension cells retained on a filter membrane on a multilayer microchip consisting of poly(dimethylsiloxane) (PDMS). ${ }^{13}$ Therefore, we selected a PDMS polymer for the fabrication of the microfluidic chip. Also, since in the conventional bioassay using a well or microchip, only one assay can be done by a series of the procedures, it is not suitable for the multiple assays. However, there is a greater demand for simultaneous assay handled with multiple samples, e.g., the assay for the optimum concentration of a drug.

In this study, a microbioassay system that realizes an anticancer agent test using animal cells, i.e., cell culturing, stimulation, and determination, was developed. To realize the simultaneous and efficient bioassay under the multiple concentrations of the agent, a simple gradient mixer was developed and attempted to integrate into the microbioassay system. The developed gradient mixer allows the gradient dilution of the solution by simply flowing two solutions on a microchip using laminar flow and diffusion in a microspace. This microfluidic system demonstrates a simultaneous bioassay using living cells in the gradient concentrations of the agent solution.

\section{Experimental}

\section{Reagents and materials}

The poly(dimethylsiloxane) (PDMS) elastomer (Sylpot 184) was obtained from Dow Corning Asia (Tokyo, Japan). A 


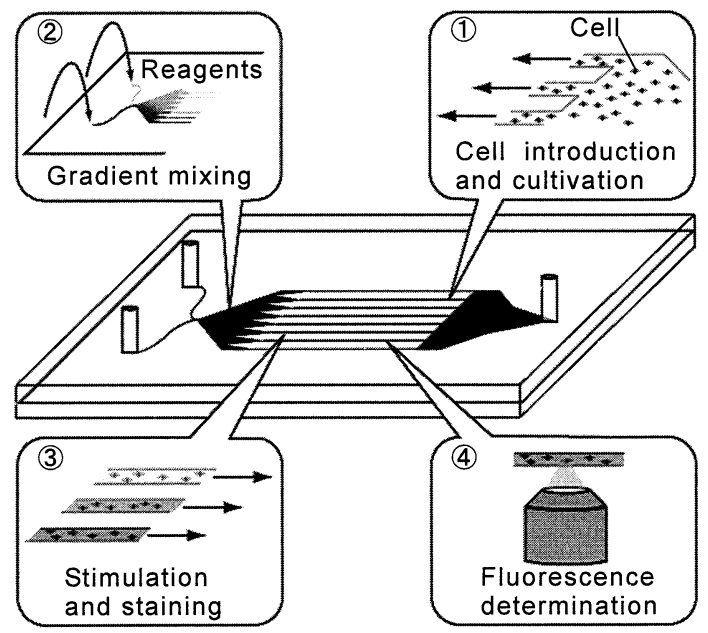

Fig. 1 Schematic image of the microbioassay system.

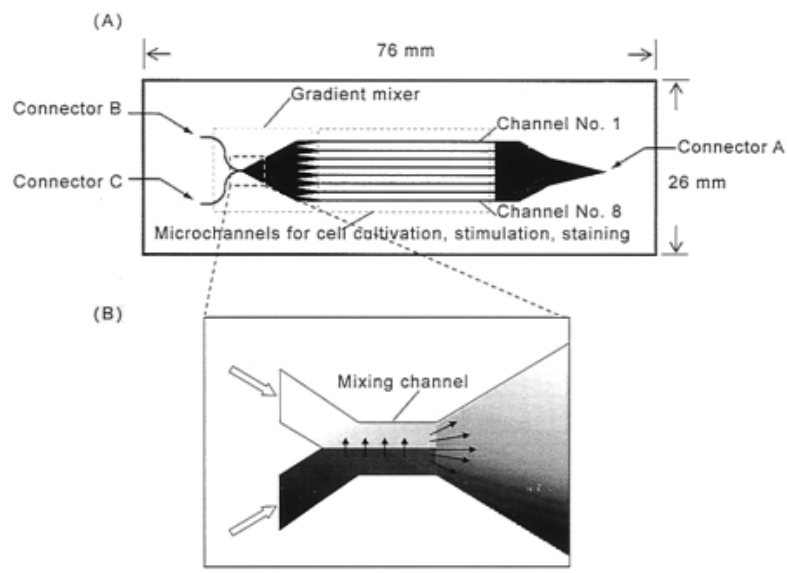

(C)

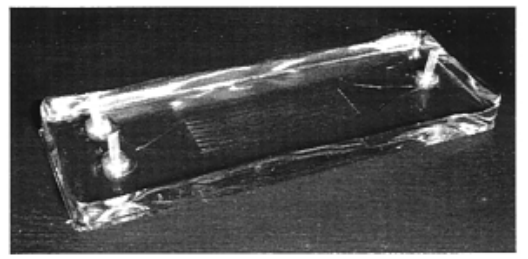

Fig. 2 (A) Illustration of the microbioassay chip, (B) principle of the gradient mixer and $(\mathrm{C})$ photograph of the microchip.

negative photoresist, SU-8 2100, was obtained from MicroChem Corp. (Newton, MA). Fibronectin was purchased from Asahi Techno Glass Corporation (Chiba, Japan). 5Fluorouracil (5-FU) and fluorescein were purchased from Wako Pure Chemical Industries, Ltd. (Osaka, Japan). EthD-1 and phosphate-buffered saline (PBS) were obtained from Invitrogen Japan K. K. (Tokyo, Japan). The stomach cancer cell line SH10-TC was obtained from the Institute of Development, Aging and Cancer, Tohoku University (Miyagi, Japan), and cultured at $37^{\circ} \mathrm{C}$ with $5 \% \mathrm{CO}_{2}$ in RPMI-1640 medium supplemented with penicillin, streptomycin, and $10 \%$ fetal bovine serum.

\section{Apparatus}

On-chip fluorescence detection was conducted using a fluorescence microscope, an inverted research microscope,
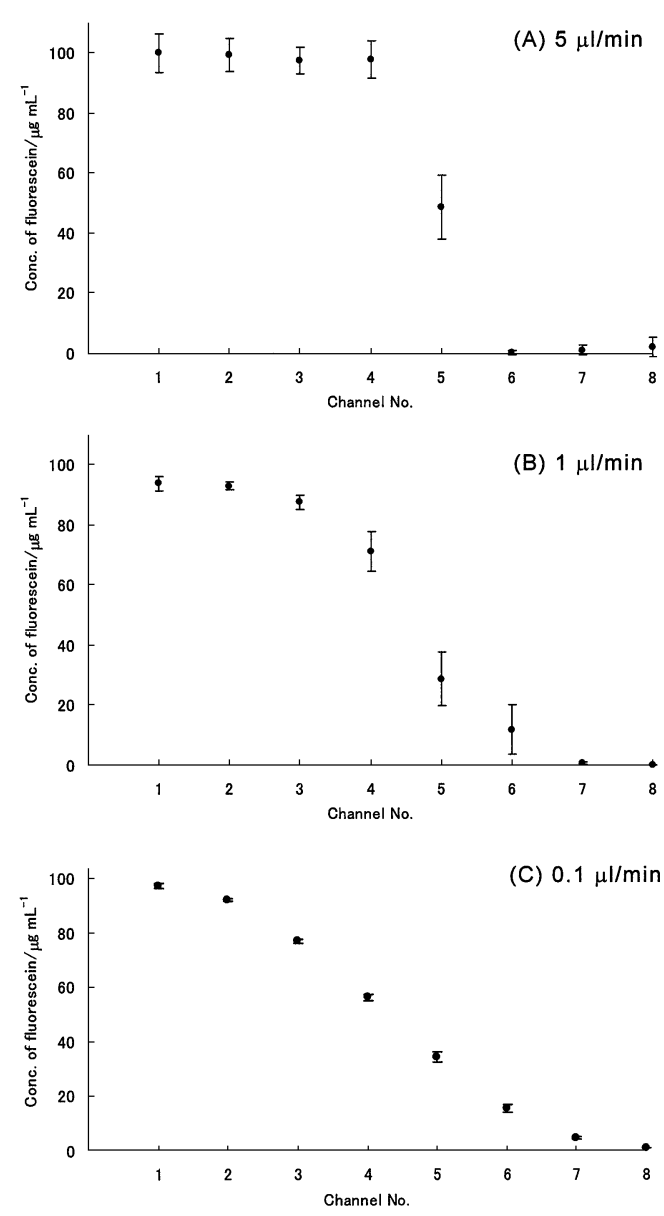

Fig. 3 Concentration of fluorescein in each microchannel. Flow rate: (A) $5 \mu \mathrm{l} / \mathrm{min}$; (B) $1 \mu \mathrm{l} / \mathrm{min}$; (C) $0.1 \mu \mathrm{l} / \mathrm{min}$.

IX71 (Olympus), and accessories. The excitation lamp was a 100-W mercury lamp U-LH100HGAPO. A filter cube, UMWIBA2, with an excitation filter (BP 460-490 nm), a dichroic mirror (DM $505 \mathrm{~nm}$ ), a barrier filter (BA 510-550 $\mathrm{nm}$ ), and a $\times 10$ objective lens (UPlan Apo $\times 10$ ) were used for the detection of fluorescein. In the case of the detection of EthD-1, a filter cube, U-MWG2, with an excitation filter (BP $510-550 \mathrm{~nm}$ ), a dichroic mirror (DM $570 \mathrm{~nm}$ ), and a barrier filter (BA $590 \mathrm{~nm}$ ) was used. A thermal incubation box, IBMU (Olympus), was mounted on the microscope to incubate the whole system. A photonic multichannel spectral analyzer, PMA-11 (Hamamatsu Photonics), was connected to the microscope, and the fluorescence of fluorescein at $550 \mathrm{~nm}$ was measured (time of exposure: $0.5 \mathrm{~s}$ ).

\section{Microchip fabrication}

The microbioassay system is illustrated in Fig. 1. All processes required for the bioassay, i.e., cell culture, agent stimulation, and determination of cell viability, were integrated into the microchip to realize the assay on the chip. The design of the microchip is shown in Fig. 2. The microchip consisted of a gradient mixer, microchannels, and three connectors (A to C). The microchannels were used for cell culturing, stimulation, and fluorescence determination. The microchip was composed of two PDMS sheets $(26 \times 76 \mathrm{~mm})$, i.e., bottom and cover plates 1 and $4 \mathrm{~mm}$ thick, respectively. The cover plate included the three connectors: the outlet (inner diameter of $1 \mathrm{~mm}$ ), the medium inlet, and the agent (each of the latter two with an inner 

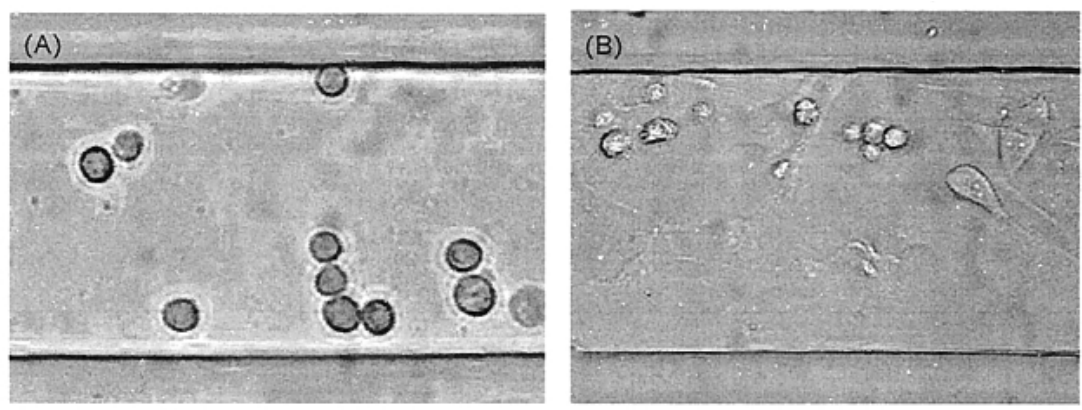

Fig. 4 (A) Micrographs of SH-10-TC cells in the microchip immediately after the introduction and (B) after $24 \mathrm{~h}$ in culture.

diameter of $250 \mu \mathrm{m})$. The outlet was also used for cell introduction. The cover plate also had a gradient mixer and microchannels for cell culturing, stimulation, and determination, which were designed using Illustrator 10 (Adobe). PDMS microchips were fabricated using usual soft-lithographic techniques..$^{14,15}$ First, a negative photoresist (SU-8 2100) was microfabricated on a glass plate (master template). Next, the PDMS pre-polymer was cast on this template. After being heated $\left(70^{\circ} \mathrm{C}, 1 \mathrm{~h}\right)$ and peeled from the template, this PDMS plate was bonded with a flat-bottom PDMS plate using oxygen plasma to form a channel structure using an oxygen plasma reactor PR301 (Yamato).

\section{Gradient mixing in a microchip}

The microchip had a function which made eight gradients from two fluids. Figure 2 shows the microchip design for an anti-cancer agent test. Eight channels $(200 \mu \mathrm{m}$ wide $)$ were connected to the mixing channel which had two inlets. The mixing channel, which intersected two inlet channels, was 200 $\mu \mathrm{m}$ wide, and the microchannel depth was $100 \mu \mathrm{m}$. The length and maximum width of gradient mixer from mixing channel to branches were set for 8 and $10 \mathrm{~mm}$, respectively. The principle of the gradient mixer is shown in Fig. 2B. First, liquids with and without reagent are introduced into the microchannel continuously from each inlet. At this time, the liquids are formed laminar flow, and beginning a diffusion of the reagent across the interface of the two solutions. Next, at the boundary between mixing channel and the following broadened microchannel, the laminar flow is gradually stretched by the spreading flow profile. Consequently, the reagent is gradually diluted, and separated into each microchannel. To determine the gradient of the solution, the culture media with and without $100 \mu \mathrm{g} / \mathrm{ml}$ of fluorescein were fed from connectors B and C, respectively. The solutions were fed from syringes set on a syringe pump KDS 230 (KD scientific) via fused-silica capillary tubes $(100-\mu \mathrm{m}$ i.d., $200-\mu \mathrm{m}$ o.d.). The flow rate of both solutions was set at the same rate, varying from 0.1 to $5 \mu \mathrm{l} / \mathrm{min}$. The fluorescence of fluorescein was measured in each microchannel using a fluorescence microscope.

\section{Cell culture on a microchip}

The microchip was washed with $70 \%$ ethanol and PBS, successively. The microchannels were coated with $5 \mu \mathrm{g} / \mathrm{ml}$ fibronectin in PBS. Next, the microchip was placed in the thermal incubation box $\left(37^{\circ} \mathrm{C}\right)$ of the microscope, and then the microchannels were rinsed with a culture medium. SH-10-TC cells were suspended with the culture medium, and $10 \mu \mathrm{l}$ of the suspension $\left(1 \times 10^{3}\right.$ cells $\left./ \mu \mathrm{l}\right)$ was introduced into the inlet connecting tube (A) using a micropipette. The tube was connected to a syringe pump via a capillary tube, and a culture medium flowed to carry the cells to the microchannels at 0.1 $\mu \mathrm{l} / \mathrm{min}$ for $24 \mathrm{~h}$

\section{Anti-cancer agent test on a microchip}

The cell viability assay was carried out on the microchip using 5-FU as an anti-cancer agent and EthD-1 as a cell-staining agent. 5-FU has been one of the most effective chemotherapeutic options available for the treatment of stomach cancers. ${ }^{16,17}$ EthD-1 penetrated non-living cells, which were counted using a fluorescence microscope. After the cells were cultured on the chip, culture media with and without 5-FU flowed via connectors B and C, respectively, at $0.1 \mu \mathrm{l} / \mathrm{min}$ for $24 \mathrm{~h}$. Finally, EthD-1 in a culture medium was reconnected to connector $\mathrm{B}$, the non-living cells were counted, and the cell viability was calculated in each channel. To confirm the fluidity in the microchannel and the effect of the anti-cancer agent, two levels of the concentration $(100$ and $10 \mathrm{mg} / \mathrm{ml})$ of the agent were attempted. In this examination, the microchip was single use only.

\section{Results and Discussion}

\section{Gradient mixing in a microchip}

First, we tested whether agents could actually be diffused and separated into each microchannel. The fluorescence of fluorescein, diluted using the gradient mixer, was measured on the microchip. Figure 3 shows the concentration of fluorescein in each microchannel. The gradient of the fluorescein observed in each channel was dependent on molecular diffusion. When the flow rate was higher than $1 \mu \mathrm{l} / \mathrm{min}$, sufficient diffusion was not observed in the microchannel (Figs. 3A snd B). However, when the flow rate was $0.1 \mu \mathrm{l} / \mathrm{min}$, an almost linear gradient of fluorescein was observed. Eight gradient concentrations of the fluorescein solution, ranging from 1 to $98 \mu \mathrm{g} / \mathrm{ml}$ (RSD $<10 \%, n$ = 3), were obtained at $0.1 \mu \mathrm{l} / \mathrm{min}$ on the microchip (Fig. 3C). These results show that effective dilution in the microspace can be carried out simply by flowing two solutions using this gradient mixer and microchannels.

\section{Cell culture on a microchip}

Figure 4 shows micrographs of SH-10-TC cells in the microchannel. When the cell suspension was fed from connector A with the medium, the cell suspension spread throughout the entire microchannel (Fig. 4A). To attach the cells to the microchannel, the flow rate was set at low velocity $(0.1 \mu \mathrm{l} / \mathrm{min})$. A micrograph of the cells after being cultured for $24 \mathrm{~h}$ on the microchip is shown in Fig. 4B. The shapes of cells 
(A)

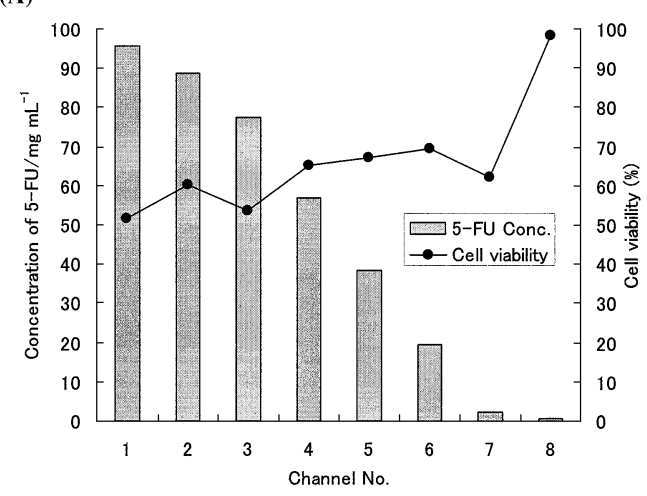

(B)

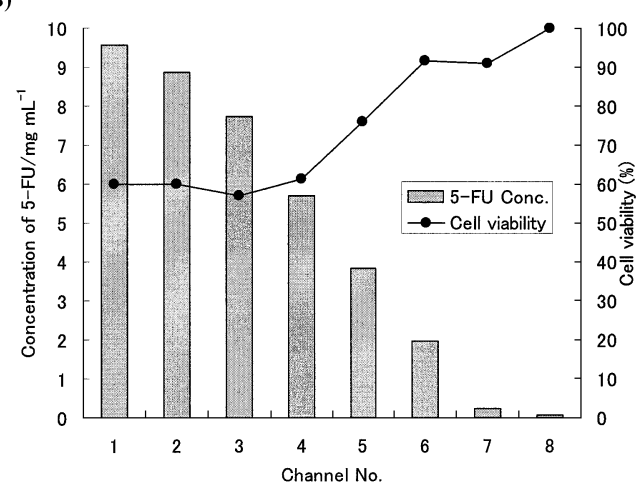

Fig. 5 Results of anti-cancer agent test on the microchip with gradient concentrations of 5-Fluorouracil in each channel. Initial concentration of the agent: (A) $100 \mathrm{mg} / \mathrm{ml}$; (B) $10 \mathrm{mg} / \mathrm{ml}$. •, cell viability; bar, concentration of 5-FU.

had changed after their introduction, and attachment of the cells was observed.

\section{Anti-cancer agent test on the microchip}

The anti-cancer agent test on the microchip with gradient concentrations of 5-FU in each channel was performed, and the results are shown in Fig. 5. Eight gradient concentrations of the 5-FU solution, ranging from 0.1 to $9.8 \mathrm{mg} / \mathrm{ml}$ and 1 to 98 $\mathrm{mg} / \mathrm{ml}$, were estimated at $0.1 \mu \mathrm{l} / \mathrm{min}$ on the microchip. When the concentration of the agent was $100 \mathrm{mg} / \mathrm{ml}$ (Fig. 5A), the borderline of the effect of 5-FU was hard to confirm. However, when the concentration was $10 \mathrm{mg} / \mathrm{ml}$ (Fig. 5B), cell viability changed according to the estimated concentration of 5-FU, and the minimum concentration of 5-FU which affected the cancer cells was estimated at about $4 \mathrm{mg} / \mathrm{ml}$. As shown in the results, the microbioassay system can complete the anti-cancer test using live cells simultaneously in eight individual channels on a microchip. The calculated reproducibility between the microchip was fewer than $10 \%$. The reaction time can be controlled by the flow rate of each reagent, and the concentration range can be regulated by a change in the agent concentration and channel number. Moreover, the gradient mixer can be applied to combinatorial chemical reactions, drug screening, and calibration.

\section{Conclusions}

All processes of the assay, i.e., cell culturing, chemical stimulation of cells, cell staining, fluorescence determination, and observation of cells, were successfully integrated on a microchip. Stomach cancer cells (SH-10-TC) were cultured successfully in the microchannels. Eight gradient concentrations of the fluorescein solution, which ranged from 1 to $98 \mu \mathrm{g} / \mathrm{ml}$, were obtained on the microchip equipped with the gradient mixer, and the microchip was applied to an anti-cancer agent test using animal cells. Simultaneous assay on these cultured cells using the gradient concentration of the anti-cancer agent was demonstrated on the microchip. This simultaneous gradient assay was conducted easily by simply flowing two fluids in microchannels. The results of this assay show that the optimum concentration of the anti-cancer agent can be estimated at $4 \mathrm{mg} / \mathrm{ml}$ of 5 -FU.

The proposed microbioassay system can be used to evaluate various effects of a sample solution on cellular responses. It is possible to use this system for many other bioanalyses using cells, including drug screenings and other assays. The microchip-equipped gradient mixer has a novel potential as an analytical system for screening and for the combinatorial reaction of chemical compounds.

\section{Acknowledgements}

This research was supported by Grant-in-Aid for Scientific Research from Japan Society for the Promotion of Science, Japan and the Nanotechnology Project of the Ministry of Agriculture, Forestry and Fisheries of Japan.

\section{References}

1. P. A. Auroux, D. Iossifidis, D. R. Reyes, and A. Manz, Anal. Chem., 2002, 74, 2637.

2. T. H. Park and M. L. Shuler, Biotechnol. Prog., 2003, 19, 243.

3. Y. Matsubara, Y. Murakami, M. Kobayashi, Y. Morita, and E. Tamiya, Biosens. Bioelectron., 2004, 19, 741.

4. M. Goto, K. Sato, A. Murakami, M. Tokeshi, and T. Kitamori, Anal. Chem., 2005, 77, 2125.

5. M. Tokeshi, T. Minagawa, and T. Kitamori, Anal. Chem., 2000, 72, 1711.

6. H. Hisamoto, T. Horiuchi, M. Tokeshi, and T. Kitamori, Anal. Chem., 2001, 73, 1382.

7. H. Hisamoto, T. Saito, M. Tokeshi, A. Hibara, and T. Kitamori, Chem. Commun., 2001, 24, 2662.

8. M. Ueno, H. Hisamoto, T. Kitamori, and S. Kobayashi, Chem. Commun., 2003, 8, 936.

9. M. Yamada, V. Kasim, M. Nakashima, J. Edahiro, and M. Seki, Biotechnol. Bioeng., 2004, 88, 489.

10. M. Yamada, M. Nakashima, and M. Seki, Anal Chem., 2004, 76, 5465.

11. S. Fujii, T. Tokuyama, M. Abo, and A. Okubo, Analyst, 2004, 129, 305.

12. S. Fujii, T. Tokuyama, M. Abo, and A. Okubo, Anal. Sci., 2004, 20, 209.

13. T. Tokuyama, S. Fujii, K. Sato, M. Abo, and A. Okubo, Anal. Chem., 2005, 77, 3309.

14. D. C. Duffy, J. C. McDonald, O. J. A. Schueller, and G. M. Whitesides, Anal. Chem., 1998, 70, 4974.

15. J. W. Hong, K. Hosokawa, T. Fujii, M. Seki, and I. Endo, Biotechnol. Prog., 2001, 17, 958.

16. Y. Jin, D. E. Heck, G. DeGeorge, Y. Tian, and J. D. Laskin, Cancer Res., 1996, 56, 1978.

17. M. Azuma, T. Yamashita, K. Aota, T. Tamatani, and M. Sato, Biochem. Biophys. Res. Commun., 2001, 282, 292. 